\title{
System Design and Dynamic Walking of Humanoid Robot KHR-2
}

\author{
Jung-Yup Kim, Ill-Woo Park, Jungho Lee, Min-Su Kim, Baek-kyu Cho and Jun-Ho Oh \\ Mechanical Engineering \\ Korea Advanced Institute of Science and Technology \\ 373-1 Guseong-dong, Yuseong-gu, Daejeon 305-701, South Korea \\ Kirk1@mclab3.kaist.ac.kr,Junhoh@ohzlab.kaist.ac.kr
}

\begin{abstract}
In this paper, we describe the mechanical design, system integration and dynamic walking of the humanoid, KHR-2 (KAIST Humanoid Robot-2). KHR-2 has 41 DOFs in total, that allows it to imitate various human-like motions. To control all joint axes effectively, the distributed control architecture is used, which reduces computation burden on the main controller, and allows convenient system. A servo motor controller was used as the sub-controller, whereas a 3-axis force/torque sensor and an inertia sensor were used in the sensory system. The main controller attached on the back of KHR-2 communicates with the sub-controllers in real-time through CAN (Controller Area Network) protocol. Windows XP was used as the operation system, whereas RTX HAL extension commercial software was used to realize the real-time control capability in Windows environment. We define the walking pattern and describe several online controllers in each stage. Some of the experimental results of KHR-2 are also presented.
\end{abstract}

Index Terms - Humanoid robot, KHR-2, Dynamic Walking

\section{INTRODUCTION}

The research of humanoid robot is diverging into the various categories such as the artificial intelligence, robot hardware development, realization of biped locomotion and human-robot interaction. As these researches make progress many researchers have started to make their focus on the human friendly robots, which is partially inspired by the rapid growth of technology. In the past, robots were confined to the factory as manipulators that were used such tasks as welding and parts-assembly in automobiles and electronic devices. Their objectives, specification and design parameters were well defined within a framework based on economics, productivity and efficiency. Now the desired functions of robots have changed, and continue to change. Robots are expected to perform various functions such as walking, speaking, recognizing, etc.

Since recently, many researches have been working on developing of humanoid biped robot, which is similar to a human being. The Honda humanoid [1, 2], the WABIAN series of Waseda University [6, 7], H6 and H7 [3, 4], HRP [5] and JOHNNIE [8] are well known human scale humanoid biped robots. Since the humanoid biped robot is complicated, expensive and unstable system, it is very difficult to realize a real-time motion control based on the sensory feedback in order to make it move the way human moves.

The objective of this research is to develop a reliable humanoid robot which is capable of accommodating and implementing various theories and algorithms such as dynamic walking, human-robot interaction, AI (Artificial Intelligence), visual \& image recognition, navigation, etc. The major objective of this work is to develop the platform and to realize dynamic walking. We used Windows OS (operating system), which is familiar to many software developers today so that it would be helpful to implement and maintain the system. The mechanical parts have simple shapes which can be machined by $2-\mathrm{D}$ process and the electrical parts are easy to upgrade, replace and reprogram. The actuator requirements including power, torque, speed, etc., have already been studied in KHR-0 [10]. KHR-0 which was developed in 2001 has 2 legs without the upper body. We studied the actuator requirements using the robot. We designed, including the size change, a new version of KHR series robot based on KHR-1 [9]. In detail, KHR-2 has updated design in the mechanical and electrical architecture. The joint stiffness and the movable joint angle ranges have been improved, and its appearance has become more human-like, and human friendly.

\section{SYSTEM DESIGN}

\section{A. Design Philosophy}

The design concept is described as follows.

1) Human like shape and movement

2) Light weight, compact size and backlash free actuator

3) Self-contained system

4) Kinematical simple structure

5) Low power consumption

According to above design concept, we designed a child-sized robot which resembles a human shape and has sufficient joints to imitate human motion (Fig. 1). The selection of child-sized human like robot came from the concerns of practicality, power efficiency, and humanfriendliness. DC motors and harmonic drive gears were 
used in the actuators. DC motors are relatively compact,

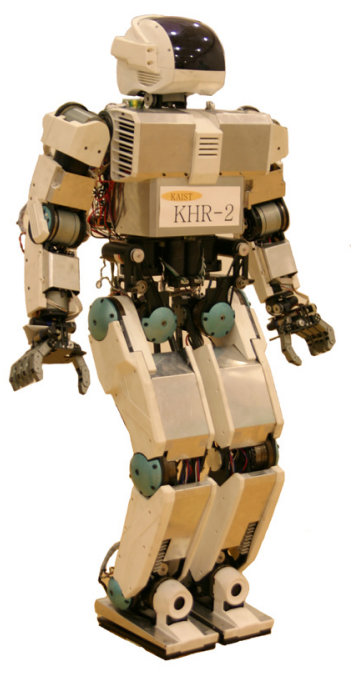

Fig. 1 The KHR-2 Humanoid

and easy to control, whereas harmonic gears help reducing backlash. All controllers, sensory devices and batteries etc. are built-in to KHR-2, while operations are performed by notebook PC using wireless LAN. In order to reduce the power consumption, the shape and thickness of the mechanical structure have been optimized. We also designed axes of all main joints to cross at one point that leads to a simple closed-form solution of inverse kinematics.

\section{B. Overview of KHR-2}

KHR-2 has 41 DOFs and its height and weight are $120 \mathrm{~cm}$ and $54 \mathrm{Kg}$, respectively. Its body was mostly made of aluminum. The specifications and DOFs are given in Table I and II. Fig. 2 shows joint structure of KHR-2.

TABLE I

SPECIFICATION OF KHR-2

\begin{tabular}{|c|c|c|}
\hline \multicolumn{2}{|l|}{ Height } & $120 \mathrm{~cm}$ \\
\hline \multicolumn{2}{|l|}{ Weight } & $54 \mathrm{~kg}$ \\
\hline \multicolumn{2}{|c|}{ Waking speed } & $0 \sim 1.0 \mathrm{~km} / \mathrm{h}$ \\
\hline \multicolumn{2}{|c|}{ Step period } & $0.9 \sim 1.0 \mathrm{sec}$ \\
\hline \multicolumn{2}{|c|}{ Grasping force } & $0.5 \mathrm{~kg} /$ finger \\
\hline \multicolumn{2}{|c|}{ Actuator } & $\begin{array}{l}\text { DC servo motor + Harmonic drive } \\
\text { gear/planetary gear }\end{array}$ \\
\hline \multicolumn{2}{|c|}{ Control Unit } & $\begin{array}{l}\text { Walking control unit, } \\
\text { Motor control unit, } \\
\text { Data transmission unit }\end{array}$ \\
\hline \multicolumn{2}{|c|}{ Sensory devices } & $\begin{array}{l}\text { 3-axis Force/Torque sensor (wrist, } \\
\text { foot) } \\
\text { Rate Gyro/Acceleration sensor } \\
\text { (trunk) } \\
\text { CCD camera (eye) }\end{array}$ \\
\hline \multirow{2}{*}{$\begin{array}{l}\text { Power } \\
\text { supply }\end{array}$} & Battery & Ni-MH (24V/6.6AH, 12V/9.9AH) \\
\hline & External & $12 \mathrm{~V}, 24 \mathrm{~V}$ \\
\hline
\end{tabular}

\begin{tabular}{|r|r|}
\hline & Power \\
\hline Operation devices & $\begin{array}{l}\text { Keyboard and mouse } \\
\text { Notebook PC with wireless LAN }\end{array}$ \\
\hline
\end{tabular}

TABLE II

DEGREE OF FREEDOM OF KHR-2

\begin{tabular}{|l|l|l|}
\hline \multirow{2}{*}{ Head } & Eye & 2 DOF $\times 2=4$ DOF \\
\cline { 2 - 3 } & Neck & 2 DOF \\
\hline \multirow{3}{*}{ Arm } & Shoulder & 3 DOF $\times 2=6$ DOF \\
\cline { 2 - 3 } & Elbow & 1 DOF $\times 2=2$ DOF \\
\hline \multirow{2}{*}{ Torso } & Wrist & 2 DOF $\times 2=4$ DOF \\
\cline { 2 - 3 } & Finger & 1 DOF $\times 10=10$ DOF \\
\hline \multirow{3}{*}{ Leg } & Hip & 1 DOF \\
\cline { 2 - 3 } & Knee & 3 DOF $\times 2=6$ DOF \\
\cline { 2 - 3 } & Ankle & 1 DOF $\times 2=2$ DOF \\
\hline Total & & 41 DOF $\times 2=4$ DOF \\
\hline
\end{tabular}

\section{Upper body design}

The upper body of KHR-2 consists of head, arms, hands and torso that include 29 DOFs in total. In the upper body, it is important to consider the space because main controller, sub controllers, sensors, batteries and power supply switch panel must be installed inside the upper body. A compact structure of torso was designed with a sufficient stiffness. In detail, there is a ' $\square$ ' shaped main frame as a chest and it is supported by two thick columns that connect the upper body and lower body. In the head two CCD cameras function as eyes, and they produce a NTSC output. The CCD image capturing runs by the frame grabber at a frequency of $15 \mathrm{fps}$. Pan and tilt mechanism has been applied to eyes and neck. In most joint axes, pulley/belt drive mechanism was used because of large movable range and space. In the hands, there is one DOF in each finger. Each finger is composed of three parts and these parts are connected to each other by pulley and belt mechanism so that if the first part moves, other parts also move simultaneously with same speed. A force/torque sensor is attached to the wrists to read the forces/torques when KHR-2 handles objects, or interact with people such as hand-shaking.

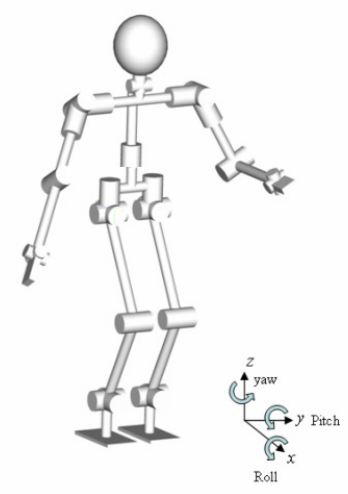

Fig. 2 Joint structure of KHR-2 
Ni-MH batteries were used to power the KHR-2 that includes the main computer, frame grabber, CAN Module, CCD cameras, sub-controllers and fans. The power consumptions of KHR-2 are 5 6A of $12 \mathrm{~V}$ and $6 \sim 10 \mathrm{~A}$ of $24 \mathrm{~V}$, so we can operate KHR-2 for about 50 minutes.

For autonomous walking, we use wireless LAN to operate KHR-2. In Windows XP, it is easy to access the main computer onboard KHR-2 by a notebook PC through wireless LAN, and then control the robot remotely.

\section{Lower body design}

It is very important to design lower body properly as the most important feature of KHR-2 is bipedal locomotion. Especially, preventing the friction of the actuator and torsion of the driving axis are important concerns because they directly affect driving efficiency. Therefore we use tension adjustment in pulley/belt mechanism and avoid the cantilever type design of driving axis. That is, all driving axis must be supported at both ends using bearings. Besides, during walking, hip joints are affected by complicated external loads periodically. Therefore, dual angular contact bearings were used in the driving axis to make it robust against external loads.

In bipedal walking, movable range of the leg and capacity of the actuator are also important issues. Table III, and IV describe the movable ranges and actuator capacities. All reduction gears of the lower body are harmonic drive gears that are free of backlash. In particular, we use two DC motors in parallel at knee pitch joint because joint torque is highest at knee during walking.

TABLE III

MOVABLE RANGE OF LOWER BODY

\begin{tabular}{|c|c|c|}
\hline \multirow{3}{*}{ Hip } & Joint & Movable angle range \\
\cline { 2 - 3 } & Roll & -90 to $+38^{\circ}$ \\
\cline { 2 - 3 } & Pitch & $-90^{\circ}$ to $+90^{\circ}$ \\
\hline \multirow{2}{*}{ Knee } & Yaw & $-77^{\circ}$ to $+60^{\circ}$ \\
\hline \multirow{2}{*}{ Ankle } & Pitch & $0^{\circ}$ to $+150^{\circ}$ \\
\cline { 2 - 3 } & Roll & $-40^{\circ}$ to $+23^{\circ}$ \\
\hline & Pitch & $-90^{\circ}$ to $+90^{\circ}$ \\
\hline
\end{tabular}

TABLE IV

ACTUATOR TYPE OF LOWER BODY

\begin{tabular}{|c|c|c|c|}
\hline & & Power transmission type & Motor Power \\
\hline \multirow{3}{*}{ Hip } & Roll & \multirow{6}{*}{$\begin{array}{c}\text { Harmonic drive gear }+ \\
\text { Pulley/Belt }\end{array}$} & $24 \mathrm{~V} / 150 \mathrm{~W}$ \\
\hline & Pitch & & $24 \mathrm{~V} / 150 \mathrm{~W}$ \\
\hline & Yaw & & $24 \mathrm{~V} / 90 \mathrm{~W}$ \\
\hline \multicolumn{2}{|c|}{ Knee } & & $24 \mathrm{~V} / 300 \mathrm{~W}$ \\
\hline \multirow{2}{*}{ Ankle } & Roll & & $24 \mathrm{~V} / 90 \mathrm{~W}$ \\
\hline & Pitch & & $24 \mathrm{~V} / 90 \mathrm{~W}$ \\
\hline
\end{tabular}

\section{SYSTEM INTEGRATION}

\section{A. Distributed Control System}

When we developed KHR-1 in 2002, we adopted the centralized control system. So a main computer with MSDOS (Microsoft Disk Operating System) controlled all control joints by using several interface cards that we developed. In this manner, we could do rapid system integration because we were able to access hardware of the main computer directly by using turbo $\mathrm{C}$ language as the thread scheduler of MS-DOS was deterministic. However it was difficult to extend the system in terms of control joints or sensors and there was a heavy calculation burden on the main computer as well. Therefore, in KHR-2, we adopted the distributed control system with Windows OS environment because KHR-2 has twice as many joints as of KHR-1 and many peripheral devices such as vision system, wireless LAN and CAN module, etc. By using distributed control architecture, calculation burden on the main computer was decreased in the expense of having to develop sub-controllers and communication bus lines between main computer and sub controllers. In addition, we have to realize real-time capability in Windows environment because Windows OS is not a real-time OS. Figure 3 illustrates the overall system configuration.

\section{B. Main Controller}

We use a commercial single board computer (PCM9575, Advantech Co.) as the main controller because it has various peripheral interfaces, easy and fast programming environment and also a good graphic user interface (GUI). Selecting criterions are fast CPU speed, low power consumption, compact size and expansion interface. CPU performance of the main computer is about Pentium III $500 \mathrm{MHz}$. and its power consumption is only 19 Watt.

\section{Communication}

The communication bus line between the main computer and the sub controllers is the means for the main computer to transmit instructions to sub controllers, or to receive data. The communication speed should be fast enough to handle 19 sub controllers concurrently. Therefore we use CAN (Controller Area Network) protocol, which assures a high speed serial communication up to $1 \mathrm{Mbit} / \mathrm{s}$. In CAN protocol, just two lines are needed for data transmission, therefore, it is very simple to extend to other sub controllers.

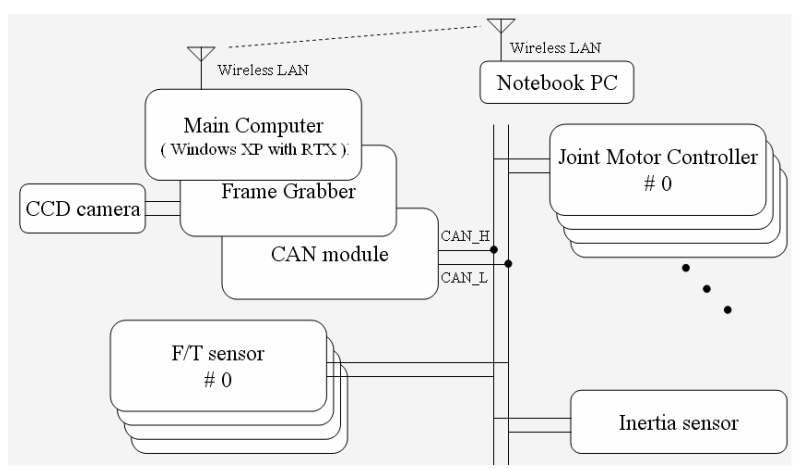

Fig. 3 Overall system configuration of KHR-2

The most important feature of CAN is its Multi- 
Master/Multi-Slave feature. This means that all controllers connected with the CAN bus line can assumes itself a master, so they can transmit any data to CAN bus line. And then, all controllers can receive data in CAN bus at the same time. So if the main controller sends the data to the CAN bus line, every controller can receive the data at the same time.

\section{Sub Controllers}

There are two kinds of sub controllers; the joint motor controller (JMC), and the inertia sensor (Fig.4 6). We developed all sub controllers and their MPUs (Micro Processor Units) to be identical. This MPU has a CAN module and communicates with a main computer. Each controller also has several A/D converters, so we can easily add sensors to them. Table $\mathrm{V}$ shows the specification of sub-controllers.

\section{E. Realization of Real-Time Control in the Main Controller}

Windows XP is a general purpose operating system (GPOS), and it is not a real-time operating system (RTOS). Therefore, it does not allow user-mode application programs to access hardware directly. The thread scheduler in Windows is not deterministic, and its interrupt latency may exceed $5 \mathrm{msec}$.

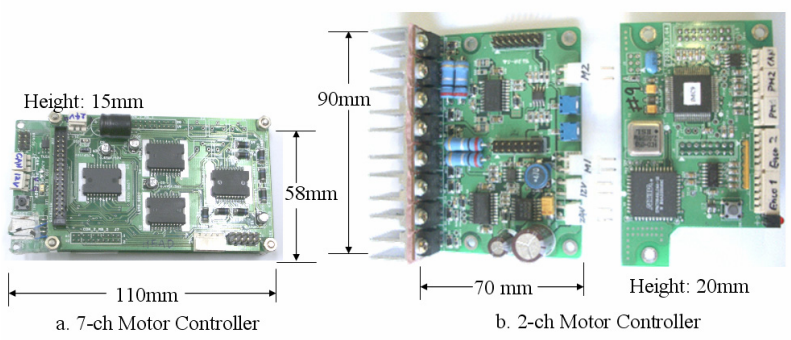

Fig. 4 Joint motor controller

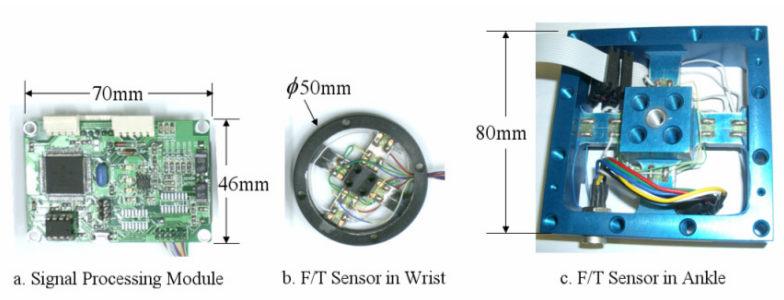

Fig. 5 F/T sensor module

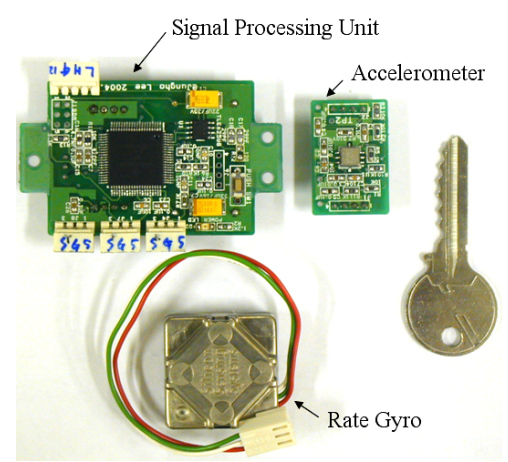

Fig. 6 Inertia sensor module
TABLE V

SPECIFICATION OF SUB-CONTROLLERS

\begin{tabular}{|c|c|c|}
\hline \multirow[t]{2}{*}{ JMC } & $\begin{array}{c}\text { Type } 1 \\
\text { (head, hand) }\end{array}$ & $\begin{array}{l}\text { CAN Module } \\
16 \text { bit micro controller } \\
7 \text { ch DC motor driver }(48 \mathrm{~W} / \mathrm{ch}) \\
5 \mathrm{ch} \text { A/D converter } \\
2 \mathrm{ch} \text { Digital output } \\
\end{array}$ \\
\hline & Type 2 & $\begin{array}{l}\text { CAN Module } \\
16 \text { bit micro controller } \\
2 \text { ch DC motor driver }(400 \mathrm{~W} / \mathrm{ch}) \\
2 \mathrm{ch} \text { A/D converter }\end{array}$ \\
\hline \multicolumn{2}{|c|}{ 3-Axis F/T sensor } & $\begin{array}{l}\text { CAN Module } \\
16 \text { bit micro controller } \\
1 \text { normal force (up to } 1000 \mathrm{~N} \text { ), } \\
2 \text { moments (up to } 30 \mathrm{Nm} \text { ) } \\
\text { Strain gage amp circuit } \\
\text { Auto balancing function } \\
\end{array}$ \\
\hline \multicolumn{2}{|c|}{ Inertia sensor } & $\begin{array}{l}\text { CAN Module } \\
16 \text { bit micro controller } \\
7 \mathrm{ch} \text { A/D converter } \\
(2 \mathrm{ch} \text { for } 2 \text {-axis acceleration sensor } \\
2 \mathrm{ch} \text { for } 2 \text {-axis rate gyro sensor } \\
1 \mathrm{ch} \text { for temperature sensor }) \\
\text { Measurable range }:-15 \sim 15 \text { deg in each axis }\end{array}$ \\
\hline
\end{tabular}

On the other hand, the control frequency of the main controller is $100 \mathrm{~Hz}$ so that we can not use Windows timer. To solve this problem we used the RTX(Real Time Extension) which is a HAL(Hardware Abstraction Layer) extension commercial software. By using RTX program, we can access hardware directly. The RTX allows making an interrupt with highest priority, which has maximum latency of only 12 usec. Using RTX we provided the realtime capability to the main computer easily, and we were able to develop the main control program quickly in Windows environment by using Visual $\mathrm{C}++$ language.

\section{MOTION CONTROL}

KHR-2 has 41 DOF and 14 joint motor controllers. Each joint motor controller controls two or seven DC motors using encoder feedback. Since KHR-2 has the distributed control architecture, the main computer sends the reference position data to the joint motor controllers constantly in exactly $10 \mathrm{msec}$ intervals (the system control frequency is $100 \mathrm{~Hz}$ ). This frequency allows sufficient calculating time for the main computer. On the other hand, the joint motor controller divides the reference position into ten time slices because the control frequency of the joint motor control is $1 \mathrm{kHz}$ (Fig. 7). In this manner, the main controller sends the reference position data to 14 joint motor controllers successively in $10 \mathrm{msec}$ intervals. Also the main controller receives the sensor data in $10 \mathrm{msec}$ intervals.

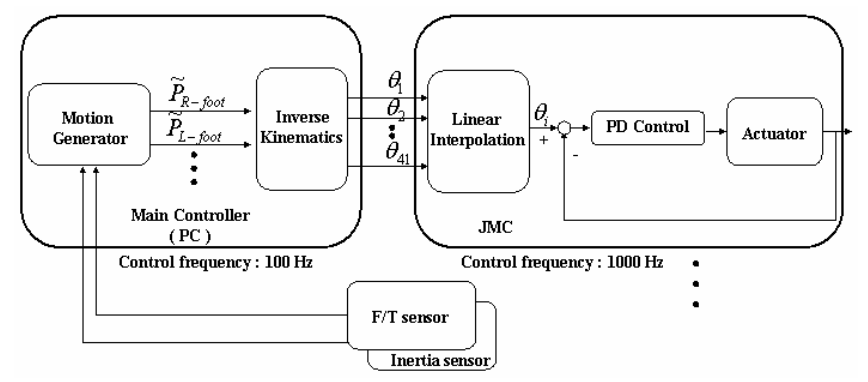

Fig. 7 Motion control 


\section{WALKING PATTERN}

The walking cycle of five stages is illustrated in Fig. 8. During walking, these stages are repeated continuously. At each stage, there is a prescribed walking motion, which is modified continuously by the motion controller. Each stage is described in detail as follows:

1) $1^{\text {st }}$ and $3^{\text {rd }}$ Stages: This stage is completely single support phase. In this stage, damping control based on torque feedback [11] is applied at the ankle to eliminate the sustained vibration of the robot.

2) $2^{\text {nd }}$ and $4^{\text {th }}$ Stages: In this stage, single support phase is changed into double support phase. So damping control is switched to landing orientation and timing control by landing detection. Landing orientation controller uses torque integral to achieve soft landing. And landing timing controller prevents the unstable landing by modification of the position schedule, when the actual landing occurs before or after the prescribed time.

3) $5^{\text {th }}$ Stage: This stage is completely double support phase. So this stage is stable. But, to keep the balance, the ZMP (Zero Moment Point) is controlled so that it is located the geometric center position between two feet.

4) $1^{\text {st }} \sim 5^{\text {th }}$ Stages: In all stages, torso roll/pitch controller is applied. The ankle pitch angles and pelvis position (in $\mathrm{x}-\mathrm{y}$ world coordinate frame) are modified to prevent the inclination of the upper body by using an inertia sensor located at the base of the torso.

Fig. 9 shows the prescribed pelvis and foot trajectories. It is known that step period is $0.95 \mathrm{sec}$ and lateral swing amplitude of pelvis is $0.64 \mathrm{~m}$. The stride is $0.4 \mathrm{~m}$ and foot elevation is $0.4 \mathrm{~m}$. All trajectories were made by using cosine and cycloid functions.

\section{WALKING EXPERIMENT}

By using the above prescribed walking pattern and online controllers, we tested the dynamic walking of KHR2. Overall strategy of walking control is described in Fig. 10. Walking experiment was performed on a floor surface, which is not perfectly flat. Fig. 11 shows measured ZMP and normal force. It is evident that ZMP trajectory does leave out of the stable region which is $80 \%$ of geometric boundary of two feet. Therefore KHR-2 can walk without falling. That is, stable walking could be accomplished.

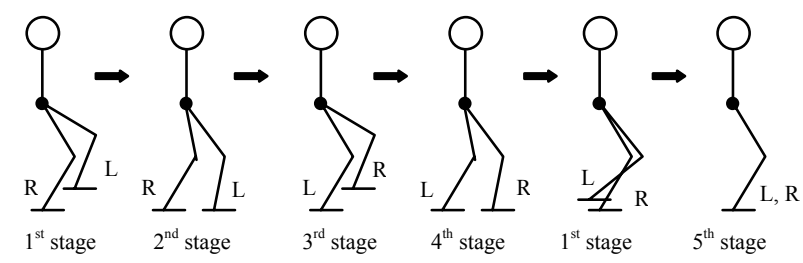

Fig. 8 Walking stages
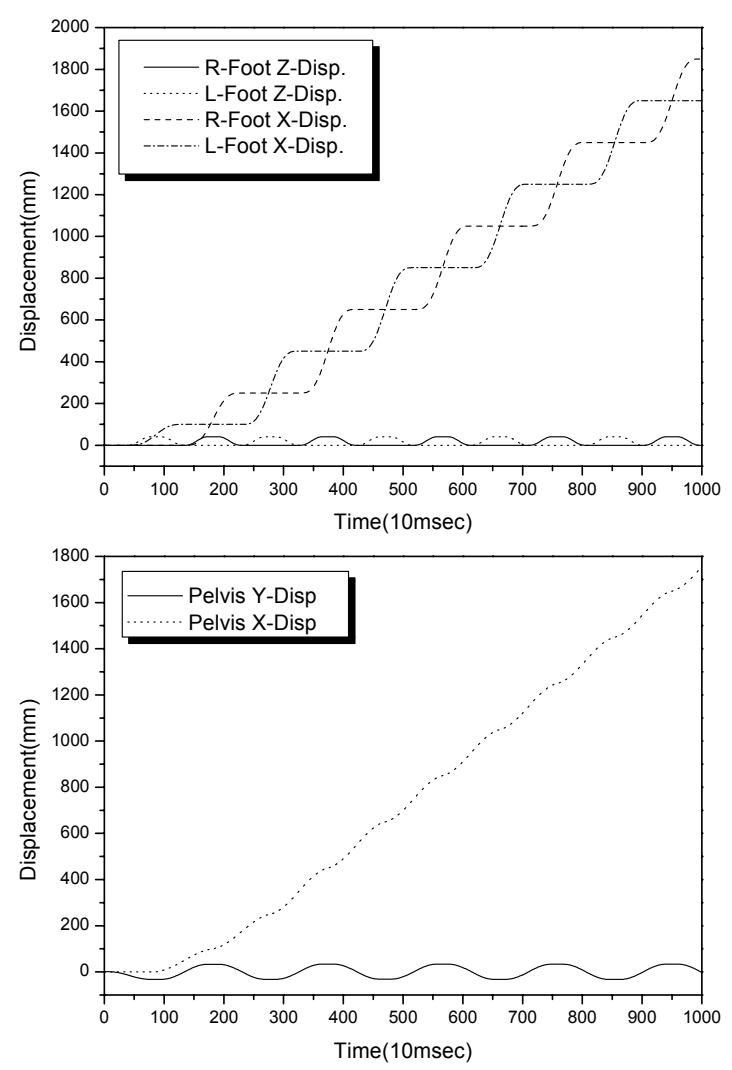

Fig. 9 Motion patterns of pelvis and foot during walking

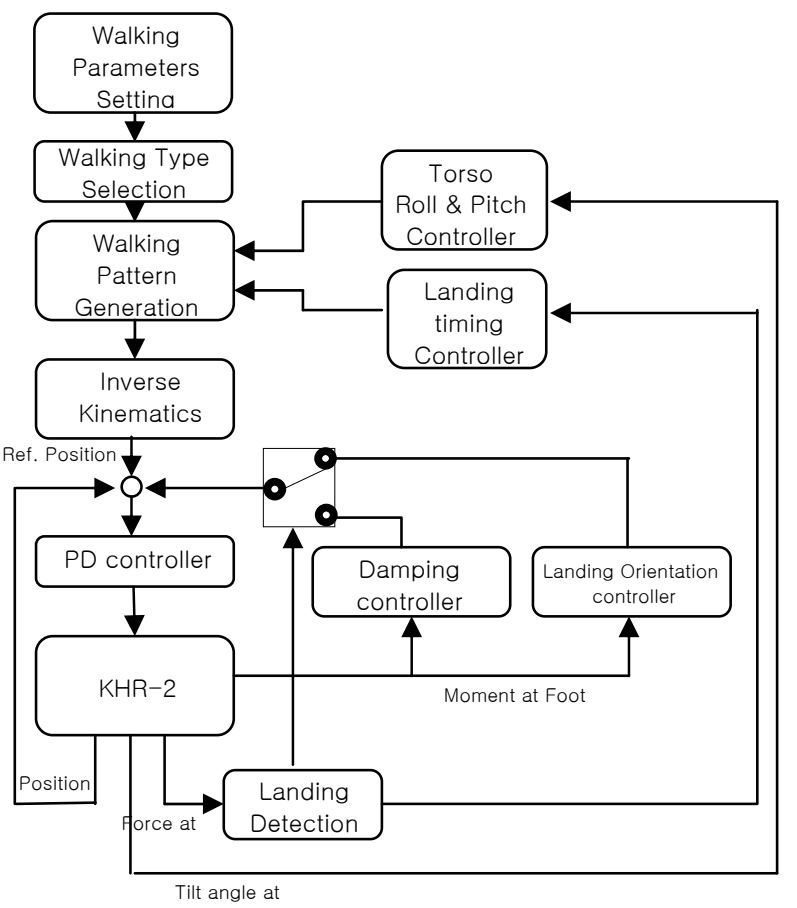

Fig. 10 Block diagram of dynamic walking control 

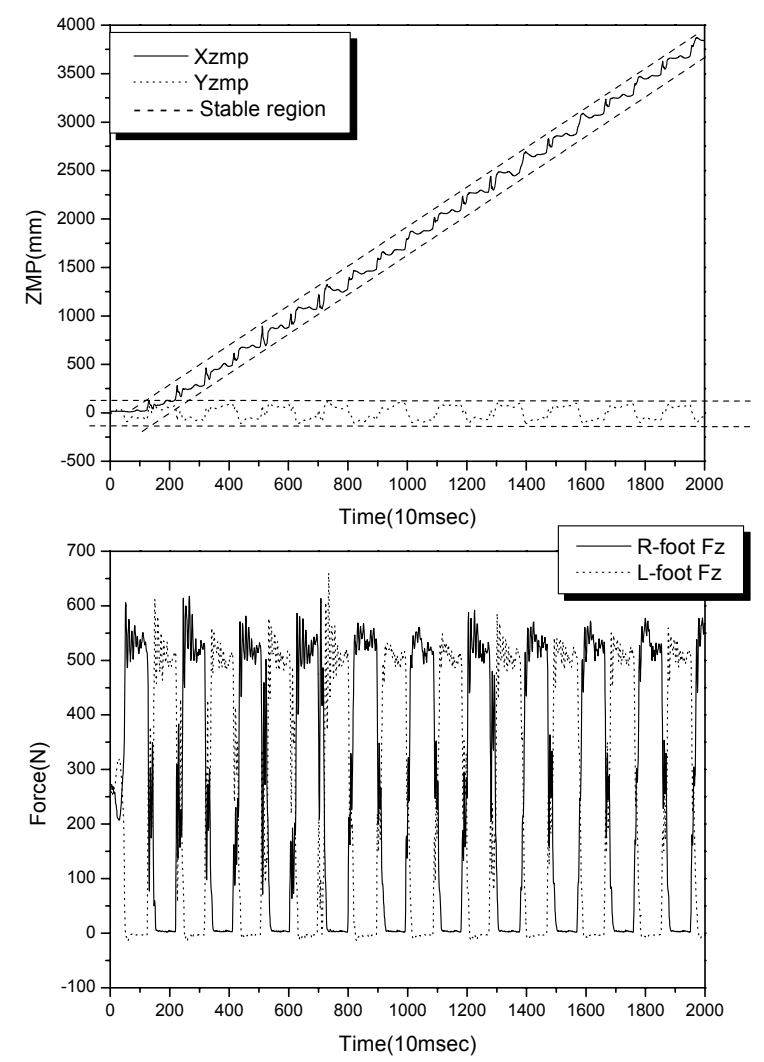

Fig. 11 Measured ZMP and normal forces on foot

\section{CONCLUSION}

The design platform of KHR-2 humanoid robot was presented. The distributed control framework has been adopted in order to control all joints and sensors efficiently. A single board computer that assures such features as fast computational speed, low power consumption, compact size and good expansion interface was used as the main controller. RTX HAL extension commercial program was used to realize the real-time capability on Windows OS environment. We also developed joint motor controllers, 3 -axis force/torque sensors and inertia sensors. The CAN protocol, which assures adequate speed was used for the serial communion between the main computer and sub controllers, as well as for real-time control.

The motion control method and walking stages were defined. In each stage, several online controls were introduced briefly. Also overall dynamic walking control algorithm was shown. In this manner, the performance of KHR-2 has been verified by walking on a not-perfectly flat floor.

\section{ACKNOWLEDGMENT}

This research is mainly supported by KAIST (Korea Advanced Institute of Science and Technology) and partly supported by HWRS (Human Welfare Robotic System),
IRRC (Intelligent Robot Research Center) and BK-21 (Brain Korea - 21) project.

\section{REFERENCES}

[1] K. Hirai, M. Hirose, Y. Haikawa, and T. Takenaka, "The Development of Honda Humanoid Robot," in Proc. IEEE Int. Conf. on Robotics and Automations, pp.1321-1326, 1998.

[2] Y. Sakagami, R. Watanabe, C. Aoyama, S. Matsunaga, N. Higaki, and K. Fujimura, "The intelligent ASIMO: System overview and integration," in Proc. IEEE/RSJ Int. Conf. on Intelligent Robots and Systems, pp. 2478-2483, 2002.

[3] K. Nishiwaki, T. Sugihara, S. Kagami, F. Kanehiro, M. Inaba, and H. Inoue, " Design and Development of Research Platform for Perception-Action Integration in Humanoid Robot: H6," in Proc. IEEE/RJS Int. Conf. on Intelligent Robots and Systems, pp.15591564,2000

[4] S. Kagami, K. Nishiwaki, J. J. Kuffner Jr., Y. Kuniyoshi, M. Inaba and $\mathrm{H}$. Inoue, "Online 3D Vision, Motion Planning and Biped Locomotion Control Coupling System of Humanoid Robot : H7," in Proc. IEEE/RSJ Int. Conf. on Intelligent Robots and Systems, pp. 2557-2562, 2002

[5] K. Kaneko, F. Kanehiro, S. Kajita, K. Yokoyama, K. Akachi, T. Kawasaki, S. Ota, and T. Isozumi," Design of Prototype Humanoid Robotics Platform for HRP," in Proc. IEEE Int. Conf. on Inteligent Robots and Systems, pp.2431-2436, 1998.

[6] H. Lim, Y. Kaneshima, and A.Takanishi, "Online Walking Pattern Generation for Biped Humanoid Robot with Trunk," in Proc. IEEE Int. Conf. on Robotics \& Automation, pp 3111-3116,2002

[7] J. Yamaguchi, A. Takanishi, and I. Kato, "Development of a biped walking robot compensating for three-axis moment by trunk motion, "in Proc. IEEE/RSJ Int. Conf. on Intelligent Robots and Systems, pp.561-566,1993.

[8] M. Gienger, K. Loffler, and F. Pfeiffer, "Towards the Design of Biped Jogging Robot," in Proc. IEEE Int. Conf. on Robotics and Automation, pp.4140-4145, 2001.

[9] J. H. Kim, S. W. Park, I. W. Park, and J. H. Oh, "Development of a Humanoid Biped Walking Robot Platform KHR-1 Initial Design and Its Performance Evaluation-," in Proc. of 3rd IARP Int. Work. on Humanoid and Human Friendly Robotics, pp.1421, 2002.

[10] J. H. Kim, I. W. Park, and J. H. Oh, "Design of Lower Limbs for a Humanoid Biped Robot," International Journal of Human friendly Welfare Robotic System, Vol.2,No.4,pp.5-10,2002.

[11] J. H. Kim and J. H. Oh, "Walking Control of the Humanoid Platform KHR-1 based on Torque Feedback Control," IEEE International Conference on Robotics \& Automation, WM-5, 2004. 